\title{
Clinical features of coronavirus disease 2019 (COVID-19) in a cohort of patients with disability due to spinal cord injury
}

\author{
Miguel Rodríguez-Cola ${ }^{1}$ Irena Jiménez-Velasco ${ }^{1}$ Francisco Gutiérrez-Henares ${ }^{2}$ Elisa López-Dolado $\mathbb{1}^{2}$ • \\ Claudia Gambarrutta-Malfatti ${ }^{1} \cdot$ Eduardo Vargas-Baquero $^{2} \cdot$ Ángel Gil-Agudo $^{2}$
}

Received: 9 April 2020 / Revised: 26 April 2020 / Accepted: 30 April 2020

(c) The Author(s), under exclusive licence to International Spinal Cord Society 2020

\begin{abstract}
Study design Cohort study of patients with spinal cord injury (SCI).

Objectives To describe the clinical and analytical features of a coronavirus disease 2019 (Covid-19) infected cohort with SCI to enable accurate diagnosis and to outline prevention measures.

Setting This study was conducted at the National Hospital for Paraplegics (Toledo, Spain).

Methods A cohort analysis of seven patients with SCI infected by Covid-19 was performed. Diagnosis was confirmed with reverse transcriptase polymerase chain reaction (RT-PCR) of nasal exudate or sputum samples. Clinical, analytical, and radiographic findings were registered.

Results RT-PCR detected COVID-19 infection in all patients, affecting males and people with a cervical level of injury more often (five out of seven). The average delay for diagnostic confirmation was 4 days (interquartile range, 1-10). Fever was the most frequent symptom (six out of seven). The second most common symptom was asthenia (four out of seven), followed by dyspnea, cough, and expectoration (three out of seven for each symptom). The Modified Early Warning System score for Covid-19 severity rating was classified as severe in five out of seven cases. All but one patient showed radiological alterations evident in chest X-rays at the time of diagnosis. All patients recovered gradually.

Conclusion Our patients with SCI and Covid-19 infection exhibited fewer symptoms than the general population. Furthermore, they presented similar or greater clinical severity. The clinical evolution was not as pronounced as had been expected. This study recommends close supervision of the SCI population to detect early compatible signs and symptoms of Covid-19 infection.
\end{abstract}

\section{Introduction}

In late December 2019, an epidemic of severe acute respiratory syndrome coronavirus 2 (SARS-CoV-2) began in Wuhan and spread rapidly throughout the world [1]. The World Health Organization declared the 2019 coronavirus disease (Covid-19) pandemic on March 11, 2020 and as of April 2, 2020, a total of 1,002,159 cases were confirmed worldwide with 51,485 deaths [2]. The first confirmed

Ángel Gil-Agudo

amgila@sescam.jccm.es

1 Department of Internal Medicine, Hospital Nacional de Parapléjicos de Toledo, Toledo, Spain

2 Department of Rehabilitation, Hospital Nacional de Parapléjicos de Toledo, Toledo, Spain imported case of Covid-19 was detected in Spain in La Gomera (Canary Islands) on January 31, 2020 [2]. Since then and up to April 2, 2020, a total of 110,286 cases have been confirmed in Spain, including 10,096 deaths [2]. On March 17, 2020, the first case of Covid-19 was confirmed at the Hospital Nacional de Parapléjicos (HNP) in Toledo Spain, a 212-bed national spinal cord injury (SCI) monographic hospital classified as a long-term healthcare facility dedicated to the comprehensive rehabilitation of people with acute SCI as well as people with chronic SCI with multiple comorbidities. The source of the first patient's infection was close contact with their relative, who was also later diagnosed with Covid19. Since then, despite having isolated the infected patient in the hospital and the use of personal protective equipment against infection by both the healthcare workers and the noninfected patients in quarantine, it was inevitable that new cases of infection were also detected among patients with up to a total of seven currently confirmed as of April 7, 2020. 
SCI involves multiple neurological and systemic complications, which drastically interfere with the quality of life and life expectancy [3]. Several demographic and clinical factors associated with the more aggressive Covid-19 phenotype have been identified: male gender, age over 60 years, and the presence of previous comorbidities such as hypertension, diabetes mellitus, obesity, cardiac ischemic disease, lung disease, and immunosuppression [4]. It is reasonable to assume SCI as a high-risk comorbidity, mostly due to the presence of respiratory failure as a result of thoracoabdominal muscle weakness [5] and also because of systemic immunosuppression due to injury $[6,7]$. Suppression of the immune system after SCI is due to noradrenergic overactivation and excess glucocorticoid release via hypothalamus-pituitary-adrenal axis stimulation [8] and also to autoimmunity [9]. Both phenomena, respiratory failure and injury-induced immunosuppression, are more pronounced after cervical or high-thoracic compared with low-thoracic SCI level, which explains why pneumonia is the leading cause of mortality in SCI patients [10]. As Covid-19 is a novel viral respiratory infection, its epidemiology and clinical course among people with SCI are yet unknown. However, recently the first survey has been published which addresses COVID-19-related practices and adaptations among health care professionals working with individuals with SCI [11]. So far, only one case of Covid-19 infection with SCI has been reported [12]. Considering all the secondary complications associated with SCI, it is reasonable to expect a harsh prognosis with Covid-19 infection.

In the present study, the authors aimed to describe the clinical features of the cohort of hospitalized SCI cases with confirmed Covid-19 infection in a monographic hospital in Spain, information which is especially important for a more accurate diagnosis and to prevent future outbreaks.

\section{Methods}

\section{Participants and inclusion/exclusion criteria}

An observational prospective analysis was made of an SCI patient cohort with confirmed Covid-19 infection from March 20 up to April 4, 2020, all of whom had been previously admitted for clinical care at the national SCI monographic hospital in Spain. All of the patients had attended an inpatient treatment regime during the present SARS-CoV2 pandemic. Those patients in whom this infection could not be confirmed were excluded.

The following demographic variables were recorded in the cohort: age, sex, SCI etiology, neurological level, and severity, with the last two variables assessed in accordance with the International Standards for Neurological Classification of SCI [13].

\section{Data sources}

The main sources of data were the electronic medical records and clinical reports of each patient. Data were recorded from the history of recent physical contacts made between the confirmed case, the diagnostic time delay until reverse transcriptase polymerase chain reaction (RT-PCR) confirmation, symptom and signs assessed at onset and evolution, and the laboratory and chest X-rays. In order to avoid bias and to ensure the patients' data confidentiality, all documents were handled after removing personal or identifying data.

\section{Variables and definitions}

Covid-19 diagnostic confirmation was defined as a positive result detected using the polymerase chain reaction with RT-PCR of nasal exudate or sputum samples according to the Spanish Ministry of Health and the Castilla La Mancha Government action procedures for cases of Infection of the new coronavirus (SARS-CoV-2) [14]. A recent physical contact exposure was defined as a close contact with a family member, caregiver, or healthcare professional diagnosed with Covid-19.

Fever was defined as a temperature higher than $37.5^{\circ} \mathrm{C}$. Lymphocytopenia was defined as a lymphocyte count of $<1500$ cells per $\mathrm{mm}^{3}$. Thrombocytopenia was defined as a platelet count of $<150.000 / \mathrm{mm}^{3}$. Pneumonia was defined based on the radiological report by a hospital radiologist, classified either as normal, with consolidation in only one lobe, bilateral multilobular, or with a ground glass pattern. In addition, the presence of atelectasis and or pleural effusion was recorded [15]. Laboratory tests included complete chemical analysis including liver and kidney function assessment, electrolytes, C-reactive protein, lactate dehydrogenase (LDH), ferritin, and blood count.

The Covid-19 severity was defined using the Modified Early Warning System (MEWS) [16]. A non-severe Covid19 case was defined by an MEWS score $\leq 2$. A severe Covid19 case was defined by an MEWS score $>2$ but $<5$. A critical Covid-19 case was defined by an MEWS score $>5$. In the case of pneumonia, we included the CURB-65 score [17].

\section{Statistical analysis}

Data were expressed as mean or median \pm standard deviation as well as a percentage. As it is a case series with only seven patients included, further statistical analysis was not performed.

\section{Results}

As of April 4, 2020, seven cases of Covid-19 have been confirmed among patients with SCI admitted to the HNP. In 
two cases with an incubation period of 4 and 9 days, respectively, the source of infection was a close contact with their relatives who were also subsequently confirmed with Covid-19. In the other five cases, no clear source of infection was identified.

\section{Demographics}

The clinical profile of the cohort is shown in Table 1. The majority were male (five out of seven; $71.4 \%$ ), with an average age of 68 years (interquartile range, 34-75). A total of five out of seven patients $(71.4 \%)$ of the cohort presented with a cervical SCI that was sensorimotor complete AIS A in four out of seven cases $(57.1 \%)$. A subacute SCI was present in more than half of the patients (four out of seven), with $<3$ months' time from injury. With respect to the SCI etiology, four of the cases were traumatic, two of them were vascular, and one of them was iatrogenic. A total of four of the cases had a tracheostomy, and two of them required frequent aspirations to remove respiratory secretions before Covid-19 infection. Four patients had a history of risk factors, such as hypertension (four out of seven; 57.1\%), dyslipidemia (four out of seven; 57.1\%), obesity (three out of seven; $42.9 \%$ ), and diabetes mellitus (one out of seven; 14.3\%). Finally, three of the patients $(42.9 \%)$ were either current or previous smokers and lung disease had been previously described.

\section{Covid-19 clinical features}

The median diagnostic time delay, defined as the time period from the onset of symptoms to confirmed Covid-19 infection with RT-PCR, was 4 days (interquartile range, $1-10)$, and was $<6$ days in six out of seven $(85.7 \%)$ of the cases. Fever was presented in six out of seven $(85.7 \%)$ of the patients at the time of diagnosis confirmation. The second most common symptom was asthenia (four out of seven; $57.1 \%$ ), followed by dyspnea, cough, and expectoration (three out of seven; $42.9 \%$ for each symptom). Neurological (two out of seven; 28.6\%) and gastrointestinal (one out of seven; $14.3 \%$ ) symptoms were less common. Only two of the patients $(28.6 \%)$ presented one single symptom from onset, while other two cases presented two symptoms and three with three or more symptoms.

The MEWS score confirmed that Covid-19's infection was severe in five out of seven of the patients (71.4\%). A total of three patients required oxygen therapy, which was always applied at a low flow $(<3 \mathrm{l} / \mathrm{min})$ to achieve $\geq 98 \%$ oxygen saturation in the capillary oximetry continuous measure.

\section{Laboratory and radiological findings}

COVID-19 infection was confirmed with RT-PCR in all the cases. Laboratory tests results and radiological results are
Table 1 Clinical features of Covid-19 SCI cohort.

\begin{tabular}{|c|c|}
\hline Variable & Sample $(N=7)$ \\
\hline Age-median (IQR) year & $68(34-75)$ \\
\hline Male sex-No./total No. (\%) & 5/7 (71.4) \\
\hline \multicolumn{2}{|l|}{ SCI level } \\
\hline Cervical (number/sample (\%)) & $5 / 7(71.4)$ \\
\hline Thoracic T1-T6 (number/sample (\%)) & $1 / 7(14.3)$ \\
\hline Thoracic T7-T12 (number/sample (\%)) & 1/7 (14.3) \\
\hline Lumbosacral (number/sample (\%)) & $0 / 7(0)$ \\
\hline \multicolumn{2}{|l|}{ SCI etiology } \\
\hline Traumatic (number/sample (\%)) & 4/7 (57.1) \\
\hline Vascular (number/sample (\%)) & 2/7 (28.6) \\
\hline Iatrogenic (number/sample (\%)) & 1/7 (14.3) \\
\hline \multicolumn{2}{|l|}{ AIS Impairment Scale } \\
\hline A (number/sample (\%)) & 4/7 (57.1) \\
\hline B (number/sample (\%)) & $0 / 7(0)$ \\
\hline C (number/sample (\%)) & $2 / 7(28.6)$ \\
\hline D (number/sample (\%)) & 1/7 (14.3) \\
\hline E (number/sample (\%)) & $0 / 7(0)$ \\
\hline Tracheostomy (number/sample (\%)) & 4/7 (57.1) \\
\hline $\begin{array}{l}\text { Need to remove respiratory secretions (number/ } \\
\text { sample }(\%) \text { ) }\end{array}$ & $2 / 4(50)$ \\
\hline Contact with Covid-19 patient (number/sample (\%)) & 2/7 (28.6) \\
\hline Incubation period-media (IQR) dias & $6.5(4-9)$ \\
\hline $\begin{array}{l}\text { History of smoking (current or former) (number/ } \\
\text { sample }(\%) \text { ) }\end{array}$ & $3 / 7(42.9)$ \\
\hline \multicolumn{2}{|l|}{ Coexisting disorders (number/sample $(\%)$ ) } \\
\hline Any & $0(0)$ \\
\hline Hypertension & $4(57.1)$ \\
\hline Diabetes & $1(14.3)$ \\
\hline Dyslipidemia & $4(57.1)$ \\
\hline Obesity & $3(42.9)$ \\
\hline Cardiovascular disease & $3(42.9)$ \\
\hline Pulmonary disease & $3(42.9)$ \\
\hline Chronic renal disease & $0(0)$ \\
\hline Cancer & $1(14.3)$ \\
\hline \multicolumn{2}{|l|}{ Symptoms of Covid-19 onset (number/sample (\%)) } \\
\hline Fever & $6(85.7)$ \\
\hline Dyspnea & $3(42.9)$ \\
\hline Cough & $3(42.9)$ \\
\hline Fatigue & $4(57.1)$ \\
\hline Sputum production & $3(42.9)$ \\
\hline Confusion & $2(28.6)$ \\
\hline Gastrointestinal symptoms & $1(14.3)$ \\
\hline \multicolumn{2}{|l|}{ Modified Early Warning System (MEWS) } \\
\hline $0-2$ points(number/sample $(\%)$ & 2/7 (28.6) \\
\hline$>3$ points (number/sample $(\%)$ & 5/7 (71.4) \\
\hline \multicolumn{2}{|l|}{ CURB-65-if pneumonia } \\
\hline $0-1$ points (number/sample $(\%)$ & $1 / 5(20)$ \\
\hline$>1$ points (number/sample $(\%)$ & $4 / 5(80)$ \\
\hline
\end{tabular}

shown in Table 2. Lymphocytopenia was found in five out of seven of the patients (71.4\%), but only one of them showed thrombocytopenia. Unfortunately, ferritin values were only available in four out of seven of the cases, of which two presented normal values and the other two showed slightly elevated levels, below $1000 \mathrm{ng} / \mathrm{ml}$. None of 
Table 2 Laboratory and radiological findings.

\begin{tabular}{|c|c|}
\hline Variable & Sample $(N=7)$ \\
\hline \multicolumn{2}{|l|}{ White-cell count } \\
\hline$<4000$ per $\mathrm{mm}^{3}-$ No./total No. $(\%)$ & $0 / 7(0)$ \\
\hline 4000-10.000 per $\mathrm{mm}^{3}$ - No./total No. $(\%)$ & $6 / 7(85.7)$ \\
\hline$>10.000$ per $\mathrm{mm}^{3}-$ No./total No. $(\%)$ & $1 / 7(14.3)$ \\
\hline \multicolumn{2}{|l|}{ Lymphocyte count } \\
\hline$<1500$ per $\mathrm{mm}^{3}-$ No./total No. $(\%)$ & $5 / 7(71.4)$ \\
\hline \multicolumn{2}{|l|}{ Platelet count } \\
\hline$<150.000$ per $\mathrm{mm}^{3}-$ No./total No. $(\%)$ & $1 / 7(14.3)$ \\
\hline Median hemoglobin (IQR)—g/dl & $11.1(8.7-14.7)$ \\
\hline \multicolumn{2}{|l|}{ Other findings-No./total No. (\%) } \\
\hline C-reactive protein $>10 \mathrm{mg} / \mathrm{dl}$ & $7 / 7(100)$ \\
\hline Procalcitonin $>0.5 \mathrm{ng} / \mathrm{ml}$ & $0 / 2(0)$ \\
\hline Lactate dehydrogenase $>250 \mathrm{U} /$ liter & $0 / 7(0)$ \\
\hline Aspartate aminotransferase $>40 \mathrm{U} /$ liter & 0/7 (0) \\
\hline Alanine aminotransferase $>40 \mathrm{U} /$ liter & $0 / 7(0)$ \\
\hline Total bilirubin $>1 \mathrm{mg} / \mathrm{dl}$ & $0 / 7(0)$ \\
\hline Urea $>40 \mathrm{mg} / \mathrm{dl}$ & $0 / 7(0)$ \\
\hline Ferritin $>500 \mathrm{ng} / \mathrm{ml}$ & $2 / 4(50)$ \\
\hline \multicolumn{2}{|l|}{ Radiologic findings } \\
\hline $\begin{array}{l}\text { Abnormalities on chest radiograph-No./total } \\
\text { No. }(\%)\end{array}$ & $5 / 7(71.4)$ \\
\hline Ground-glass opacity-No./total No. (\%) & $1 / 7(14.3)$ \\
\hline Unilobular pneumonia-No./total No. (\%) & $1 / 7(14.3)$ \\
\hline Multilobular pneumonia-No./total No. (\%) & $3 / 7(42.9)$ \\
\hline
\end{tabular}

our patients showed altered levels of alanine aminotransferase, aspartate aminotransferase and LDH.

With respect to the radiographic findings, two out of seven of our patients showed no radiological alterations at diagnosis (Fig. 1a), three out of seven of them presented bilateral multilobular pneumonia (Fig. 1b), one with unilobular pneumonia (Fig. 1c), and another one with a ground glass pattern (Fig. 1d). Only one case showed pleural effusion (Fig. 1c).

\section{Clinical evolution}

All of the patients have been gradually recovering, although the evolution was slower in the older patients, who were also dependent on tracheostomy and suffered cervical AIS A SCI. No deaths were recorded.

\section{Discussion}

To the author's knowledge, this is the first work that describes the clinical profile of Covid-19 in a cohort of SCI patients. The vulnerability of people with SCI is well known [5-10]. We have found that more than half of our patients showed one or two symptoms at the moment of Covid-19 confirmation, which is lower than the symptomatic expression in other published case series [18]. We hypothesized that the Covid-19 symptoms overlapped with
Fig. 1 Representative radiological findings. a Normal $\mathrm{x}$-ray. b Bilateral multiple lobular subsegmental pneumonia. c Unilobular pneumonia with pleural effusion. d Ground glass pattern.
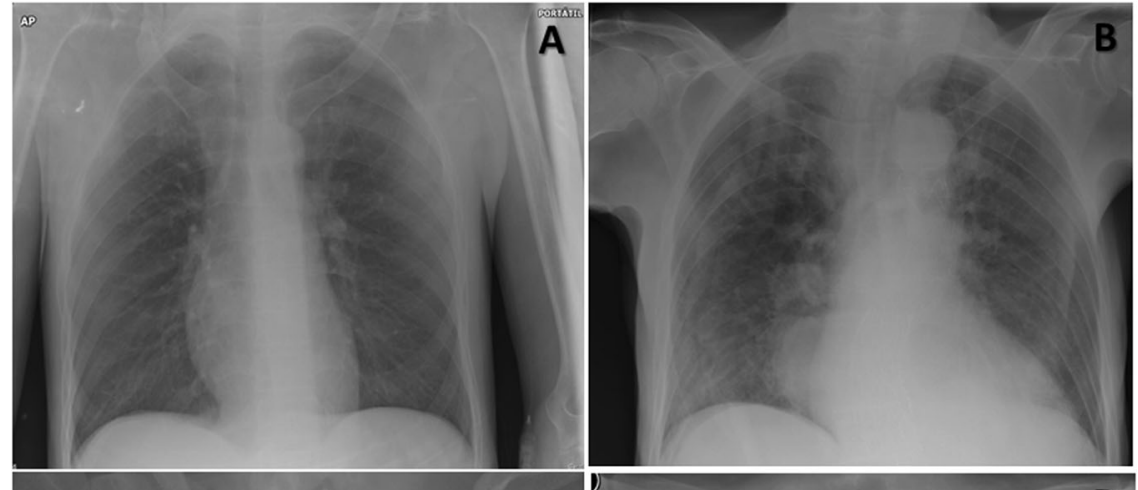

c

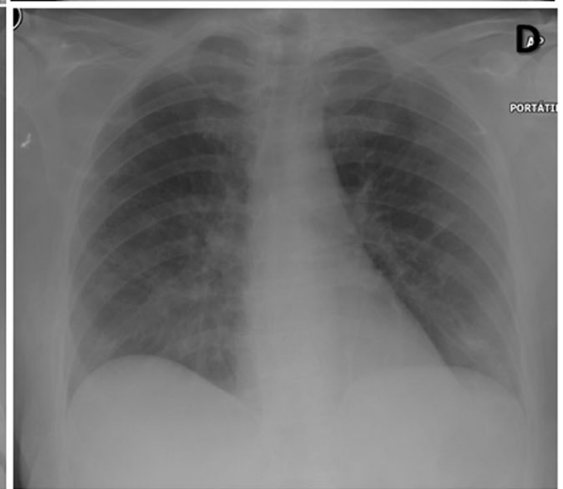


some of the signs of respiratory failure due to SCI, which would have complicated the accurate clinical diagnosis. The average time delay between diagnosis in our case series was shorter than that found in other series, which may reflect rapid early identification of the initial Covid-19 symptoms in a cohort of hospitalized patients who were already receiving close attention for their SCI. At the onset of Covid-19 infection, fever and asthenia were the most frequently observed symptoms, which slightly differed from other published series that identified cough and fever as initial symptoms in the general population [18-20]. We agree with the previous case of Covid-19 SCI in a patient with SCI which reported that cough was not the prevalent symptom [12]. However, despite the less evident symptomatology in our cohort, the MEWS score confirmed that they presented a higher risk of clinical worsening compared with the general population [18]. Before the clinical onset of Covid-19 infection, only half of the patients had a tracheostomy, which required bronchial aspiration and hyperinflation techniques to remove respiratory secretions. In contrast, after Covid-19 diagnosis, all of the patients required such techniques. In other studies, a similar progression in the requirement for respiratory assistance has also been observed for patients who require oxygen therapy [18-20], with 98\% capillary oxygen saturation achieved with low flow oxygen therapy $(<31 / \mathrm{min})$. To date, none of the patients with SCI and Covid-19 infection have required mechanical ventilation support or intensive care admission.

In agreement with most of the published studies, all of the COVID-19 cases were confirmed with RT-PCR. The radiological findings also agreed with the published data for Covid-19 infection, with the presentation of bilateral multilobe pneumonia as the most frequent radiological pattern. In addition, two-thirds of our cases also presented lymphocytopenia $[3,18,20]$. However, we did not find any alteration of transaminases or LDH levels. Serum ferritin values were obtained in almost all the patients, with only half of them showing moderately elevated levels $(<1000 \mathrm{ng} /$ $\mathrm{ml})$. These results may highlight a decreased inflammatory response in our series compared with the general population $[4,20]$ and could be consistent with a more benign evolution of Covid-19 infection for people with SCI.

We consider it of vital importance to closely supervise the SCI population to identify early compatible Covid-19 symptoms and signs, as well as to implement follow-up measures against infectious diseases contagion. These measures will be especially needed in long-term treatment facilities such as our hospital, where asymptomatic cases could become Covid-19 reservoirs, further complicating the eradication of the current epidemic or future outbreaks.

The limitations of this study include the fact that none of the patients admitted to the HNP or their healthcare personnel were interviewed for symptoms of infection or received the SARS-CoV-2 screening test, which therefore may have led to an underestimation of the Covid-19 infection rate in the hospital, especially in those patients who were asymptomatic or who presented mild symptoms.

As this is a descriptive case series study, there is no control group, the absence of which is justified given the urgency of pandemic and the lack of previous Covid-19 data among SCI population. Unfortunately, it is likely that our Covid-19 SCI case series will gradually increase until the end of the present pandemic, which will provide us with more clinical data with longer evolution times.

\section{Conclusion}

In our case series, patients with SCI and confirmed Covid19 infection exhibited fewer symptoms than the general population. Furthermore, although they presented a similar or greater MEWS severity the clinical evolution of Covid19 infection was not as pronounced as had been expected. This study recommends close active supervision of the SCI population to detect early compatible signs and symptoms of Covid-19 infection.

\section{Data availability}

The main sources of data were the electronic medical records and clinical reports of each one of the patients. The history of recent contact with a confirmed case, the diagnosis time delay, the symptom and signs of onset and evolution, the laboratory and chest X-rays were analyzed. In order to avoid biases and ensure the patients' data confidentiality, all documents were handled after removing their filiation data. The corresponding author had full access to all data in the study and had final responsibility for the decision to submit for publication.

Acknowledgements Dr Julian Taylor (JTG) revised the paper and reviewed the English version (julian.taylor@jtgconsulting.org).

Author contributions MRC was responsible for designing the clinical protocol and collecting clinical and analytical data, conducting the research, analyzing data, interpreting results, and writing the paper. IJV was responsible for designing the clinical protocol and collecting clinical and analytical data. FGH was responsible for designing the clinical protocol and collecting clinical and analytical data. ELD was responsible for designing the clinical protocol and collecting clinical and analytical data, identifying bibliographic resources, analyzing data, interpreting results, and writing the paper. CGM was responsible for designing the clinical protocol and conducting the research. EVB was responsible for designing the clinical protocol and collecting clinical and analytical data. AGA was responsible for designing the clinical protocol, reviewing data quality, conducting the research, analyzing data, interpreting results, and writing the paper. 


\section{Compliance with ethical standards}

Conflict of interest The authors declare that they have no conflict of interest.

Ethical approval We certify that all applicable institutional and governmental regulations concerning the ethical use of human volunteers were followed during the course of this research. The guidelines of the declaration of Helsinki were followed in every case and the study design was approved by the local ethics committee (Hospital Virgen de la Salud, Toledo, Spain, resolution number 504).

Informed consent All participants provided their informed consent.

Publisher's note Springer Nature remains neutral with regard to jurisdictional claims in published maps and institutional affiliations.

\section{References}

1. Li Q, Guan X, Wu P, Wang X, Zhou L, Tong Y, et al. Early transmission dynamics in Wuhan, China, of novel coronavirus-infected pneumonia. NEJM. 2020, January. https:// doi.org/10.1056/NEJMoa2001316.

2. Coronavirus COVID-19 Global Cases by the Center for Systems Science and Engineering (CSSE) at Johns Hopkins University (JHU). JHU COVID-19 Resource Center. Johns Hopkins Coronavirus Resource Center. 2020. https://www.arcgis.com/apps/ opsdashboard/index.html\#/bda7594740fd40299423467b48e9ecf6.

3. Zhou F, Yu T, Du R, Fan G, Liu Y, Lui Z, et al. Clinical course and risk factors for mortality of adult inpatients with COVID-19 in Wuhan, China: a retrospective cohort study. Lancet. 2020;395: P1054-62. https://doi.org/10.1016/S0140-6736(20)30566-3.

4. Sun X, Jones ZB, Chen XM, Zhou L, So KF, Ren Y, et al. Multiple organ dysfunction and systemic inflammation after spinal cord injury: a complex relationship. J Neuroinflammation. 2016;13:260. https://doi.org/10.1186/s12974-016-0736-y.

5. Shah A, Shem K, McKenna S, BerllyY M. Respiratory management of the spinal cord injured patients. In: Kirshblum S, Campagnolo DI, editors. Spinal cord medicine. Philadelphia: Lippincott Williams \& Wilkins; 2011.

6. Riegger T, Conrad S, Liu K, Schluesener HJ, Adibzahdeh M, Schwab JM. Spinal cord injury-induced immune depression syndrome (SCI-IDS). Eur J Neurosci. 2007;25:1743-7.

7. Kasinathan N, Vanathi MB, Subrahmanyam VM, Rao JV. A review on response of immune system in spinal cord injury and therapeutic agents useful in treatment. Curr Pharm Biotechnol. 2015;16:26-34.

8. Prüss H, Tedeschi A, Thiriot A, Lynch L, Loughhead SM, Stutte $\mathrm{S}$, et al. Spinal cord injury-induced immunodeficiency is mediated by a sympathetic-neuroendocrine adrenal reflex. Nat Neurosci. 2017;20:1549-59. https://doi.org/10.1038/nn.4643

9. Arévalo-Martin A, Grassner L, García-Ovejero D, PaniaguaTorija B, Barroso-García G, Mach O, et al. Elevated autoantibodies in subacute human spinal cord injury are naturally occurring antibodies. Front Immunol. 2018;9:2365. https://doi. org/10.3389/fimmu.2018.02365.

10. Kopp MA, Watzlawick R, Martus P, Failli V, Finkenstaedt FW, Chen $\mathrm{Y}$, et al. Long-term functional outcome in patients with acquired infections after acute spinal cord injury. Neurology. 2017;88:892-900.

11. Stillman MD, Capron M, Alexander M, Longini Di Giusto M, Scivoletto G. COVID-19 and spinal cord injury and disease: results of an international survey. Spinal Cord Ser Cases. 2020;6:21.

12. Righi G, Del Popolo G. COVID-19 tsunami: the first case of a spinal cord injury patient in Italy. Spinal Cord Ser Cases. 2020;6:22.

13. American Injury Association. International standards of neurological classification of spinal cord injury. Richmond, VA: American Injury Association; revised 2019.

14. Protocolo de actuación frente a casos de infección por el Nuevo Coronavirus (SARS-CoV-2). Ministerio de Sanidad. Gobierno de España. 2020. https://www.mscbs.gob.es/profesionales/saludPublica/ ccayes/alertasActual/nCovChina/documentos/Procedimiento_ COVID_19.pdf.

15. Zu ZY, Jiang MD, Xu PP, Chen W, Ni QQ, Lu GM, et al. Coronavirus disease 2019 (COVID-19): a perspective from China. Radiology. 2020:200490. https://doi.org/10.1148/radiol.2020200490.

16. Subbe C, Kruger M, Rutherford P, Gemmel L. Validation of a modified early warning score in medical admissions. QJM. 2001;94:521-6.

17. Lim WS, van der Eerden MM, Laing R, Boersma WG, Karalus N, Town GI, et al. Defining community acquired pneumonia severity on presentation to hospital: an international derivation and validation study. Thorax. 2003;58:377-82.

18. Guan WJ, Ni Zy, Hu Y, Liang WH, Ou CQ, He JX, et al. Clinical characteristics of coronavirus disease 2019 in China. N Engl J Med. 2020. https://doi.org/10.1056/NEJMoa2002032.

19. Fauci AS. Covid-19-navigating the uncharted. N Engl J Med. 2020;382:1268-9. https://doi.org/10.1056/NEJMe2002387.

20. Huang C, Wang Y, Li X, Ren L, Zhao J, Hu Y, et al. Clinical features of patients infected with 2019 novel coronavirus in Wuhan, China. Lancet. 2020;395:497-506. 